\title{
Language and Critical Thinking Integrated Teaching Reform on English Public Speaking Course in the Context of "New Liberal Arts"
}

\author{
Yang Chen \\ Zhejiang Yuexiu University, Shaoxing, China \\ Email: roy0901@163.com
}

How to cite this paper: Chen, Y. (2021) Language and Critical Thinking Integrated Teaching Reform on English Public Speaking Course in the Context of "New Liberal Arts". Open Access Library Journal, 8: e7535. https://doi.org/10.4236/oalib.1107535

Received: May 17, 2021

Accepted: July 4, 2021

Published: July 7, 2021

Copyright $\odot 2021$ by author(s) and Open Access Library Inc.

This work is licensed under the Creative Commons Attribution International License (CC BY 4.0).

http://creativecommons.org/licenses/by/4.0/

\section{(c) (i) Open Access}

\begin{abstract}
In the context of "New Liberal Arts and Macro Foreign Language Education", the integration of critical thinking skills into English language learning will not only contribute to the discipline development, but also meet the needs of cultivating high-quality foreign language talents in the context of the new era. As individuals' English speaking abilities are closely related to their thinking skills, teachers should pay attention to improving students' critical thinking competence while enhancing their English language proficiency and speaking skills. Based on the current teaching situation of English Pubic Speaking course, this article proposes specific strategies such as transforming teachers' role, setting up language and critical thinking integrated teaching goals, adopting diverse teaching methods, cultivating students' critical thinking literacy and disposition, as well as upgrading traditional teaching modes, in order to provide some references for subsequent experimental research.
\end{abstract}

\section{Subject Areas}

Educational Reform

\section{Keywords}

New Liberal Arts, Public Speaking, Critical Thinking, Teaching Reform

\section{1. 引言}

2018 年，我国中共中央提出了 “新工科、新医科、新农科、新文科” 等 概念; 同年，教育部决定实施 “六卓越一拔尖” 计划 2.0 , 全面推动 “四新建 设”，提高人才培养质量。“新文科”这一理念最初由美国的希拉姆学院于 2017 年提出, 意指 “对传统文科进行学科重组、文理交叉, 即把新技术融入 
哲学、文学、语言等诸如此类的课程中, 为学生提供综合性的跨学科学习” [1]。 新文科的 “新” , 更加强调的是创新的 “新”, 要求各学科找到新定位, 打 破传统学科壁垒、促进学科的交叉与融合, 从而培养创新性人才, 服务于国 家战略需要。那么, 在新文科背景下, 外语学科又面临了哪些机遇与挑战呢?

2019 年, 高教司就新文科建设层面, 对外语学科提出了新要求, 如培养 能够参与全球治理的人才; 推动中华优秀传统文化的对外传播等。吴岩司长 提出, 在当今 “新文科、大外语” 的背景下, 英语教育必须注重教学, 打造 “金课”、杜绝 “水课”、培养 “一精多专”、“一专多能” 的国际化复合 人才 $[2]$ 。就外语人才培养而言, 新文科建设不仅要注重学科知识的讲授, 更 要激发学生的创新能力和批判性思维[3]。2018 年, 高校英语专业课程改革和 《国标》明确将 “思辨能力” 列为大学生的必备能力之一，最新版的《大学 英语教学指南》(2020 版)也提出了新的教学要求, 除了对语言知识与技能、 跨文化交际能力和学习策略的要求以外, 还增加了对思辨能力的要求 $[4]$ 。事 实上, 当代大学生只有具备科学审慎的思维能力和家国情怀, 日后才能更好 地参与到全球治理、担负起对外传播中华民族优秀文化的重任。

英语演讲作为一门大学英语课程, 旨在培养学生如何面向公众群体传播 新信息、或是说服他人接受某种观点。逻辑和思维能力与个体的语言输出密 切相关, 教师在提高学生英语语言能力、演讲技巧的同时, 提升其思辨力显 得尤为重要。

\section{2. 国内外思辨教学述评}

“思辨” 来源于英语的 “critical thinking” , 国外众多学者都对其定义有 不同的阐释。1990 年, Facione 博士召集众多学者, 将 critical thinking 划分 为认知能力 (cognitive skills) 和思辨特质 (disposition), 前者是指个体的阐释、 分析、评价、推理、解释、自我调节等技能; 后者指个体具备思维开放、追 求真理、思辨自信等思辨特质 [5]。

国外关于思辨教学的研究可以追溯到教育学家杜威, 到 20 世纪 80 年代 在高等教育中蓬勃发展起来, 到了 21 世纪已被多数国家列入高等教育目标之 一。在思辨教学手段领域, Ennis [6]提出了四种有效的思辨教学手段, 即 “一 般式”、“灌输式”、“沉浸式” 和 “混合式”。对于思辨教学手段的分类 旨在厘清, 思辨能力的培养是应该作为一种特定的技能进行专门教授, 还是 与学科融合、以内容为依托来提升学生的思辨力。在二语领域, 多数研究采 用 “沉浸式” 教学手段, 如利用议会制辩论、同伴评估与反馈、合作式学习、 撰写日志等提高学生的思辨能力[7]。关于英语演讲与思辨教学的研究, 国外 学者主要聚焦在构建演讲思辨模型、利用特定的教学手段、培养演讲者的批 判性特质等 [8] [9] [10]。

国内关于思辨教学的研究在近 5 年来快速上升, 思辨教学已成为外语教 学的一个研究焦点。

从理论研究层面, 文秋芳 [11]构建了思辨能力层级理论模型, 将思辨能力 分为两个层次, 第一层次为元思辨能力, 第二层次为思辨能力, 分为认知技 能和情感特质, 是在元思辨能力的管理下发展和培养的。从实证研究层面, 
有学者运用合作式学习[12]、混合翻转课堂教学[13]、教师反馈[14]等在具体 课程中(如写作、阅读)培养学生的思辨能力。

然而, 国内关于英语演讲的研究主要聚焦在克服演讲者焦虑、如何撰写 演讲稿、如何设计先声夺人的演讲开头等。只有少量学者尝试将思辨教学与 英语演讲课程融合, 如厘清英语演讲课程涉及哪些思辨能力[15]、构建英语演 讲者的思辨技能测评方法 [16]、如何提高学生在某一类演讲中的思辨力 [17]。

综上所述, 我国对于新文科背景下的思辨英语教学, 尤其是如何将思辨 力融入到具体的英语演讲教学中, 仍然存在一定的研究空间。本文以英语演 讲教学面临的问题和挑战为切入点, 提出相应的解决策略, 力图促进学生学 科知识和思辨力的同步提升。

\section{3. 英语演讲思辨教学面临的问题和挑战}

\section{1. 学生缺乏思辨素养}

在英语演讲课堂, 不少学生缺乏思辨人文素养, 这主要体现在以下两个 方面。

首先, 学生普遍缺乏思辨自信心、或思辨动机不够。例如, 在英语演讲 课堂, 教师要求学生评价同伴的一段演讲稿, 学生的评判非常 “友好”, 通 常给出一个 “很好” 的评价, 或者给出相对高的分数。大部分学生即便与他 人意见相佐, 但因为缺乏自信心、认为自己知识储备较少, 其行为模式上也 更倾向于 “接纳” 、“认可” 他人的观点。很少有学生勇于提问、评估、质 询他人的论点, 更无法有理有据地驳斥同伴的观点、甚或教师的观点。另一 方面, 在演讲课堂, 不少学生思辨动机不够, 该类型的学生缺乏深度学习, 不在意知识的内化和思维的提升, 表现得极为 “功利” 和 “现实” 。有一部 分学生对学习本身并无兴趣、也不热衷于求知和探索真相, 更在意的是考试 成绩和教师的评分准则。

其次, 学生不擅长分析问题和逻辑推理, 在演讲课上主要体现在资料的 搜集和归类、论点的确定、演讲的论证等。比如, 学生在搜集素材阶段, 无 法准确区分事实与观点; 或倾向于使用普遍常识或二手资料, 没有系统科学 的方法收集和处理数据。再如, 学生在构建论点阶段, 无法识别自己的逻辑 谬误(logical fallacy); 在处理论点和论据的关系方面, 经常缺乏事实支撑, 或 论述的细节与论点不匹配[18]。

\section{2. 英语演讲课程评价过于单一, 思辨能力未能融入具体教学}

目前, 演讲课程的教学目标主要聚焦在克服演讲焦虑、撰写演讲稿、掌 握恰当的演讲技巧等。此外, 很多高校对该课程的考核方式过于单一, 如期 末考试的定题演讲或即兴演讲, 约占学生总成绩的 70\% - 80\%, 而剩余的 $20 \%$ - 30\%则是考察学生的出勤率、课堂表现、作业等。

在该种考核体制下, 以期末考试为主的终结型评价占了大量比重, 而以 提升学生综合能力、应用能力的形成性评价非常欠缺。一方面, 教师的教学 重点是演讲知识、技巧的讲解, 忽略学生综合素质的培养。另一方面, 测试 
的反拨作用促使学生更为关注知识的识记层面, 从而导致思辩能力长期处于 缺席状态。即便教师和学生均意识到思辨能力的重要性, 由于其未能对标到 具体的教学活动和教学考核中, 学生更为关注的是短期的、显性的学习目标, 如期末测试成绩、或某一具体的课堂表现。

\section{3. 英语演讲课堂缺乏思辨教学实操}

思辨能力并非是与生俱来的, 而是在日常学习与生活中不断习得的。以 英语演讲课堂为例, 因受限于大班教学和授课时长, 教师往往以理论讲解为 主, 之后给学生布置演讲作业。这种讲练分离的授课模式无法发挥学生的思 辨能力, 加之没有明确的思辨教学目标, 导致课堂活动无法将英语语言能力、 演讲相关知识和技巧与思辨能力相融合。在有限的课堂时间内, 英语教师会 优先处理语言、知识点传授等浅层教学任务, 无法兼顾学生思辨力的培养。

此外, 尽管教师有意识将思辨能力融入到课堂活动, 但由于学生英语水 平参差不齐, 教师很难做到学生语言技能与认知水平的同步提升。有些学生 即便展现出高阶思维能力, 但由于语言能力受限, 降低了活动参与度, 教师 也只能在具体教学中舍弃这些思辨活动。

\section{4. 教师自身思辨能力受限}

不仅学生缺乏思辨能力, 一部分教师在以往的教科研中, 更关注本学科 的发展和研修, 思辨教学意识淡漠, 且缺乏与其他专业教师的互动交流。2019 年, 张虹教授通过调研, 探究了 117 名高校英语专业教师对日常思辨教学的 认知、面临的困难与需求。研究发现, 有过半的教师反映自身思辨能力贵乏, 且缺少思辨相关的理论学习和实操练习, 无法将思辨能力与课堂教学活动进 行融合 [19]。因此, 在英语演讲课堂, 教师即便认识到思辨教学的重要性, 因 自身能力受限, 也无法设计出行之有效、真正促进学生思维发展的教学活动。

\section{5. 传统的教学方式过于单一}

根据认知负荷 (cognitive load)理论, 如果学生认知负荷较重, 在一定程度 上会影响学生的工作记忆, 从而降低课堂参与度和自我效能感。长期以来, 教师在演讲课堂多采用传统的面授方式, 教师讲授知识, 之后检验学生的学 习效果。这种传统的授课模式占用了大量的课堂时间, 仅能帮学生完成一些 低层次的认知任务, 如理解、记忆等层面, 忽视了分析、评价、创造等高阶 思维能力的培养。学生如果没有足够的先验知识做支撑, 很难就某一个主题 或课堂活动进行多维度、深层次的思考。

综上所述, 英语演讲课堂融入思辨教学刻不容缓, 笔者就上述问题提出 了以下几点建议。

\section{4. 英语演讲思辨教学改革路径}

\section{1. 在日常教学中培养大学生的思辨人文素养}

吴岩司长提出, 高校要建设新文科, 要做强大外语, 必须培养高素质外 
语人才。然而, 很多英语教师仅关注英语学科的工具性, 而忽略了人文性。 英语演讲教学中要注重专业学习与思辨人文素养结合, 教师如能将一些思辨 元素融入到课堂, 可以很好地给学生插上思辨的翅膀, 继而培养学生的思辨 倾向和思辨特质。

在演讲教学中, 如学生在搜集资料、撰写演讲稿、分析论据的过程中, 教师要引导学生学会反思、善于反思, 培养他们兼容并包、尊重事实、乐于 探索的思辨品质。在日常交流中, 教师可以有意识地引导学生, 从多个角度 去分析、评价和探索世界各国文化和思潮。思辨不是吹毛求疪, 或是毫无道 理的 “批判” , 而是在夯实自己的专业基础上, 具备科学理性的人文主义价 值观，以更宽阔的视野去认识人、社会与世界。

\section{2. 将提高思辨能力与英语演讲教学目标结合}

教学目标影响教师的教学重点、以及学生的学习导向。若要将思辨教学 与该课程达到有效的融合, 必须将思辨能力纳入具体的教学目标。

演讲活动可划分为选题、资料搜集、撰写演讲稿(构建论点、论据和论证)、 反思、预演、演讲的发表等。教师应在每一个子任务中明确思辨教学目标, 如学生的阐释、分析、推理和评价能力, 与每一个演讲阶段主题结合, 真正 将其落实到每一单元的教学设计、课堂活动及作业布置中。

以 “Opening a speech” (演讲开头)这一教学单元为例, 教师在设计课前 任务就要明确本节课的教学重难点, 设定层级式教学目标: 1) 理解演讲开头 的重要性；2）阐述有哪些不同类型的演讲开头；3）对比、评价不同类型演 讲开头的特点, 以及应用主题; 4) 根据备选主题, 创设自己的演讲开头。教 师基于学生原有的知识水平和认知能力, 在课堂讲授期间, 由浅入深, 既注 重学科知识的讲解和应用, 又明确了自己的思辨教学目标。

\section{3. 运用多种思辨教学手段, 促进 “思辨” 与 “语言学习” 的深度 融合}

在新文科背景下, 外语教学应当注重语言学习和认知发展的同步提升, 不可顾此失彼。因此, 教师可结合教学任务, 将思辨的练习融入到外语学习 中; 同时在社会建构主义的理论指导下, 设计一些合作学习活动, 通过师生 交流和生生交流, 打造学习共同体, 从多维角度刺激学生的认知发展。具体 思辨教学活动如下:

首先, 教师结合每节课的教学主题和重难点, 选择合适的课堂活动。如 利用 “苏格拉底式问询” 、辩论、探究式学习等方式, 让学生参与口语思辨 活动, 讨论不同的演讲主题适合什么样的开场白、某一演讲稿在阐明论点和 整合论据方面还能如何完善; 又如, 安排学生利用思维导图或概念图, 设计 自己的英语演讲稿大纲, 提高学生的认知和元认知能力。

其次, 根据学生的性格、已掌握的知识水平, 合理分组, 设计小组活动。 如安排小组合作撰写一份报告, 分析一段演讲的语言是否生动和准确、论点 是否鲜明、论据是否充分、演讲者运用了哪些演讲技巧等。教师尽力打造建 构主义课堂, 最大程度上促进朋辈互助和知识共享, 鼓励学生从不同的角度 
分析问题和思考问题，从而提高他们的思辨力。

再次, 教师提供演讲相关的思辨评价标准, 之后安排小组互评。以互评 演讲稿为例, 以往的同伴互评只是学生对文本作出表层的反馈, 如单词的拼 写、词组搭配、语法错误等。事实上, 一篇好的演讲稿必须逻辑清晰、层次 分明、衔接恰当。提前安排学生学习并反复操练思辨评价标准, 更能有效 “对 标” 学生的思维能力和语言能力[20]。

\section{4. 教师转变角色, 提高自身思辨能力}

新文科建设对教师也提出了新的要求, 教师不再是绝对的主导者, 应当 主动转变角色, 由以往的 “知识传授者” 转为 “知识共建者” 。在这一过程 中, 教师主要担任引导者(facilitator)的角色, 最大限度调动学生的内在动机, 帮助其由被动学习到主动探索, 完成知识和技能的内化。

其次, 教师须提高自身的思辨力。在演讲授课中，教师除教授学生必备 的演讲知识、演讲技巧外, 自身也要阅读和研修相关的思辨教学材料, 如区 分常见的逻辑谬误、研究论据的有效性、掌握科学严谨的方法等。

再次, 教师要积极参与教科研讨论, 注重跨学科学习和协作式合作。只 有不断更新自己的知识体系, 才能更好地对自己的教学进行反思和评估, 为 今后的教学提供参考。

\section{5. 优化传统授课模式, 采用线上线下的混合教学模式}

新文科背景下, 教师不仅要更新学科知识, 还要利用人工智能时代和新 技术革命带来的机遇。英语演讲本身是一门应用型课程, 教师不应只采用传 统的面授方式, 还要探索线上线下的混合教学模式，从而更好地服务于思辨 教学，具体步骤如下:

课前, 教师列出知识重点、提供精彩的演讲片段, 学生可按照自己的进 度模仿和学习, 完成知识的记忆、理解等低层次学习任务。课前扫除学生的 认知负荷, 能帮助学生获取先验知识, 继而完成课堂学习的高效输入和输出。

课中, 教师除讲授重难点知识, 要注重以学生为中心。学生不再单一被 动地接收知识, 而是积极主动地参与课堂、将所学知识运用到不同情境。在 此过程中，教师根据学生的表现，提供不同的反馈，帮助学生进行意义建构、 实现知识的内化, 提升学生的高阶思维能力。

课后, 教师引导学生积极反思, 如总结演讲技巧、撰写演讲稿或反思日 志、在线分享和讨论学术困难及解决方案, 并互相点评。在此过程中, 学生 不但提高了自己的思辨力, 还为教师提供了反馈意见, 以便教师及时调整评 估标准, 更好地评估教学成果。

\section{5. 结语}

综上所述, 语言与思辨应该相辅相成, 互为支撑, 培养学生的思辨力并 不是一蹴而就的。在新文科背景下，外语人才须具备良好的学科能力和理性 思维, 才能满足我国对高素质的复合型外语人才需求。本文以具体的演讲课 程为例, 培养学生英语语言能力的同时, 又注重学生的思辨能力和人文素养, 
同时利用新技术教学，从这一层面上呼应了 “新文科” “大外语” 的理念。

本文主要的创新之处和贡献主要是: 第一, 在新文科背景下, 促进英语 语言学习和思辨力的融合。大学英语教学不仅要注重学科知识点, 还应将思 辨元素融入到教学中, 并且注意提升学生的思辨素养和思辨特质, 提高学生 的高阶思维能力。第二, 将思辨目标对标具体课程, 促进生生协作, 利用多 种思辨教学手段打造建构主义课堂, 同时优化教学考核机制。最后, 打破教 师固有角色，教师除提高自身思辨水平外，还可利用技术赋能和新的学习空 间, 最大程度上激发学生的学习动力。例如, 教师结合线上线下的混合教学 模式，以教练 (coach) 的身份，在课前、课中和课后提供必要的支架 (scaffolding)、并实施监管措施, 提高学生自主学习和自我反思的能力。

本文在分析英语演讲教学现状后, 提出了一些改革路径, 可以为后续研 究者提供一些参考。本文的不足在于, 未能在具体教学中进行实证研究, 就 某一种思辨教学手段或特定方法对学生进行教学干预。另外, 本文并没有从 不同院校的学情、师资和学科特色专业进行分析。因此, 后续研究者可以在 自己所在院校, 开展实证研究, 从微观层面, 如线上活动的思辨元素融入和 开放型问题的设置、线下课堂多种思辨教学手段的探索、对学生作业的评价 标准和具体实施细节的操作等进行完善。可以展望的是, 随着国家对大学生 创新能力、思辨能力的重视, 以及教科研工作者的不断实践, 外语学科也会 更好地服务于国家的战略需要。

\section{基金项目}

本研究为 2020 年浙江越秀外国语学院线上线下混合式教学改革项目一一 基于行动学习法在《英语演讲》混合式教学中的构建和实施(编号 JGH2005); 2020 年浙江越秀外国语学院校级课题——新文科背景下的思辨英语教学探究 - - 以《英语演讲》课程为例(编号 N2020004)的阶段性成果。

\section{Conflicts of Interest}

The author declares no conflicts of interest.

\section{References}

[1] https://baijiahao.baidu.com/s?id=1633061954813639160\&wfr=spider\&for=pc

[2] 吴岩. 新使命 大格局 新文科 大外语[J]. 外语教育研究前沿, 2019, 2(2): 5-9, 92.

[3] 向明友. 新学科背景下大学外语教育改革刍议[J]. 中国外语, 2020, 17(1): 19-24.

[4] 何莲珍. 新文科与外语学科建设一综合性大学的探索与实践 [J]. 中国外语, 2021, 18(1): 8-9.

[5] Facione, P.A. (1990) Critical Thinking: A Statement of Expert Consensus for Purposes of Educational Assessment and Instruction. https://files.eric.ed.gov/fulltext/ED315423.pdf

[6] Ennis, R.H. (1989) Critical Thinking and Subject Specificity: Clarification and Needed Research. Educational Researcher, 18, 4-10. https://doi.org/10.3102/0013189X018003004

[7] El Soufi, N. and See, B.H. (2019) Does Explicit Teaching of Critical Thinking Im- 
prove Critical Thinking Skills of English Language Learners in Higher Education? A Critical Review of Causal Evidence. Studies in Educational Evaluation, 60, 140-162. https://doi.org/10.1016/j.stueduc.2018.12.006

[8] Wagner, P.E. (2018) Reviving Thinking in a Speaking Course: A Critical-Thinking Model for Public Speaking. Communication Teacher, 33, 158-163.

https://doi.org/10.1080/17404622.2018.1536795

[9] Sellnow, D.D. and Ahlfeldt, S.L. (2005) Fostering Critical Thinking and Teamwork Skills via a Problem-Based Learning (PBL) Approach to Public Speaking Fundamentals. Communication Teacher, 19, 33-38. https://doi.org/10.1080/1740462042000339258

[10] Akatsuka, Y. (2019) Awareness of Critical Thinking Attitudes and English-Speaking Skills: The Effects of Questions Involving Higher-Order Thinking. Journal of Pan-Pacific Association of Applied Linguistics, 2, 59-84. https://doi.org/10.25256/PAAL.23.2.4

[11] 文秋芳, 王建卿, 赵彩然, 刘艳萍, 王海妹. 构建我国外语类大学生思辨能力量 具的理论框架 [J]. 外语界, 2009(1): 37-43.

[12] 潘琳琳, 宋毅. 合作学习与思辨能力的培养[J]. 外语与外语教学, 2016, 287(2): 97-105.

[13] 李倩, 廖开洪. 基于思辨能力发展的学术英语翻转混合教学研究[J]. 天津外国语 大学学报, 2020, 27(1): 129-145.

[14] 李莉文, 刘雪卉. 教师反馈与思辨能力培养的个案研究——以英语专业本科毕业 论文为例[J]. 外语界, 2018, 189(6): 20-27.

[15] 孙旻, 俞露, 王晶. 英语演讲实践中的思辨分项技能一一以说服性演讲为例 [J]. 中国外语, 2015, 12(5): 49-56.

[16] 孙旻. 英语演讲学习者思辨技能测评方法对比研究 [J]. 外语界, 2018, 187(4): 51-58.

[17] 林岩. 英语演讲教学中的思辨能力培养——“说服策略”单元课例研究[J]. 中国外 语, 2020, 17(3): 63-69.

[18] 田朝霞. “英语演讲”课在中国高校的本土化再研讨一一形成性评估课程设计与实 施[J]. 外语教育研究前沿, 2018, 1(1): 26-34.

[19] 张虹. 思辨英语教学: 英语专业教师认知视角 [J]. 外语研究, 2019, 36(4): 57-62.

[20] 孙有中. 思辨英语教学原则[J]. 外语教学与研究, 2019, 51(6): 825-837.

\section{Appendix (Abstract and Keywords in Chinese)}

\section{新文科背景下的英语演讲思辨教学改革}

摘要: 在 “新文科、大外语” 的背景下, 将思辨力融入到英语语言学习中, 不仅有助于提升学科发展, 更符合新时代背景下培养高素质外语人才的需要。 英语演讲能力与思辨力紧密相关, 教师在培养学生英语语言能力和演讲技巧的 同时, 也要注重提高学生的思辨力。本文针对英语演讲课程的教学现状, 提出 转变教师角色、设立思辨英语教学目标、采用多元教学手段、提高学生的思辨 人文素养、优化传统教学模式等具体策略, 以期为后续的实验研究提供一些参 考。

关键词: 新文科, 公众演讲, 思辨能力, 教学改革 